7. E. Steinitz, Rechteckige Systeme und Moduln in algebraischen Zahlkörpern, Math. Ann. vol. 71 (1911) pp. 328-354, vol. 72 (1912) pp. 297-345.

8. O. Taussky, On a theorem of Latimer and MacDuffee, Canadian Journal of Mathematics vol. 1 (1949) pp. 300-302.

9. H. Zassenhaus, Neuer Beweis der Endlichkeit der Klassenzahl bei unimodularer Äquivalenz endlicher ganzahliger Substitutionsgruppen, Abhandlungen aus dem Mathematischen Seminar der Universität Hamburg vol. 12 (1938) pp. 276-288.

UNIVERSITY OF ILLINOIS

\title{
FLEXIBLE ALMOST ALTERNATIVE ALGEBRAS ${ }^{1}$
}

D. M. MERRIELL

1. Introduction. Almost left alternative algebras were defined by Albert in [1]. They are algebras $A$ over a field $F$ of characteristic not two which satisfy these postulates:

I. The elements of $A$ satisfy an identity of the form

$$
\begin{aligned}
z(x y)= & \alpha(z x) y+\beta(z y) x+\gamma(x z) y+\delta(y z) x+\epsilon y(z x) \\
& +\eta x(z y)+\sigma y(x z)+\tau x(y z)
\end{aligned}
$$

for elements $\alpha, \beta, \gamma, \delta, \epsilon, \eta, \sigma, \tau$ in $F$ which are independent of $x, y, z$ in $A$.

II. The relation $x x^{2}=x^{2} x$ holds for every $x$ of $A$.

III. There exists an algebra $B$ with a unity quantity $e$ such that $B$ satisfies (1) and is not a commutative algebra.

An algebra is called almost right alternative if I, II, and III hold with (1) replaced by an identity of the same form but with $z(x y)$ replaced by $(x y) z$. These two identities are the general shrinkability conditions of level one, as defined by Albert in [2]. An almost alternative algebra is one which is both almost left alternative and almost right alternative.

Reference is made in [1] to several results which are proved here. In addition to the above postulates, we assume the flexible law, that is, $(x y) x=x(y x)$ for every $x$ and $y$ in $A$. This makes Postulate II redundant. Albert confined his investigation in [1] to nonflexible algebras.

Received by the editors February 20, 1956.

1 This paper is a portion of a Ph.D. thesis supervised by A. A. Albert and submitted to the University of Chicago in 1951. The author is now at Robert College, Istanbul. 
It is shown in this paper that flexible almost left alternative algebras are also almost right alternative, power-associative, Jordanadmissible, and that they are quasiequivalent to alternative algebras except when the parameter $\alpha+\beta=3 / 4$. Results for the latter case will be presented in another paper. The class of flexible almost left alternative algebras includes quasiassociative algebras. It is included in the class of noncommutative Jordan algebras as defined in [3].

2. Almost left alternative algebras. From Postulate III, if we replace $x, y, z$ in (1) by $e$, we obtain

$$
\alpha+\beta+\gamma+\delta+\epsilon+\eta+\sigma+\tau=1 \text {. }
$$

Next replace only $z$ by $e$. Then $(1-\alpha-\gamma-\eta-\tau) x y=(\beta+\delta+\epsilon+\sigma) y x$ and (2) implies that $(1-\alpha-\gamma-\eta-\tau)(x y-y x)=0$. Since $B$ in Postulate III is not commutative, $x y \neq y x$ for some $x$ and $y$. Hence

$$
\alpha+\gamma+\eta+\tau=1, \quad \beta+\delta+\epsilon+\sigma=0 .
$$

Now replace $x$ by $e$. Then $(1-\alpha-\beta-\gamma-\eta)(z y-y z)=0$, and so

$$
\alpha+\beta+\gamma+\eta=1, \quad \delta+\epsilon+\sigma+\tau=0 .
$$

Finally replace $y$ by $e$. Then $(1-\alpha-\beta-\delta-\epsilon)(z x-x z)=0$, and so

$$
\alpha+\beta+\delta+\epsilon=1, \quad \gamma+\eta+\sigma+\tau=0 .
$$

From (4) and (5),

$$
\epsilon=1-\alpha-\beta-\delta, \quad \eta=1-\alpha-\beta-\gamma .
$$

From (3) and (6),

$$
\sigma=\alpha-1, \quad \tau=\beta .
$$

Hence there are only four essential parameters in (1).

The identity (1) may be expressed in terms of right and left multiplications of $A$ and is equivalent to

$$
\begin{aligned}
R_{x y}= & \alpha R_{x} R_{y}+\beta R_{y} R_{x}+\gamma L_{x} R_{y}+\delta L_{y} R_{x}+\epsilon R_{x} L_{y}+\eta R_{y} L_{x} \\
& +\sigma L_{x} L_{y}+\tau L_{y} L_{x} .
\end{aligned}
$$

Interchanging $x$ and $y$, we obtain

$$
\begin{aligned}
R_{y x}= & \alpha R_{y} R_{x}+\beta R_{x} R_{y}+\gamma L_{y} R_{x}+\delta L_{x} R_{y}+\epsilon R_{y} L_{x}+\eta R_{x} L_{y} \\
& +\sigma L_{y} L_{x}+\tau L_{x} L_{y} .
\end{aligned}
$$

If we interchange $y$ and $z$ in (1), the resulting identity is equivalent to

$$
\begin{aligned}
L_{x} L_{y}= & \alpha L_{y x}+\beta L_{y} R_{x}+\gamma L_{x y}+\delta R_{y} R_{x}+\epsilon R_{y x}+\eta L_{y} L_{x} \\
& +\sigma R_{x y}+. \tau R_{y} R_{x} .
\end{aligned}
$$


Interchanging $x$ and $y$ in (10), we obtain

$$
\begin{gathered}
L_{y} L_{x}=\alpha L_{x y}+\beta L_{x} R_{y}+\gamma L_{y x}+\delta R_{x} R_{y}+\epsilon R_{x y}+\eta L_{x} L_{y} \\
+\sigma R_{y x}+\tau R_{x} L_{y} .
\end{gathered}
$$

If we interchange $x$ and $z$ in (1), the resulting identity is equivalent to

$$
\begin{gathered}
R_{y} L_{x}=\alpha L_{x} R_{y}+\beta L_{x y}+\gamma R_{x} R_{y}+\delta L_{y x}+\epsilon L_{x} L_{y}+\eta R_{x y} \\
+\sigma R_{x} L_{y}+\tau R_{y x} .
\end{gathered}
$$

Interchanging $x$ and $y$ in (12), we obtain

$$
\begin{aligned}
R_{x} L_{y}= & \alpha L_{y} R_{x}+\beta L_{y x}+\gamma R_{y} R_{x}+\delta L_{x y}+\epsilon L_{y} L_{x}+\eta R_{y x} \\
& +\sigma R_{y} L_{x}+\tau R_{x y} .
\end{aligned}
$$

Now add equations (8) through (13). The result together with (2) is equivalent to

$$
\begin{aligned}
(\alpha+\beta+\gamma+\delta) & \left(R_{x y+y x}-L_{x y+y x}-R_{x} R_{y}-R_{y} R_{x}+L_{x} L_{y}\right. \\
& \left.+L_{y} L_{x}+R_{y} L_{x}+R_{x} L_{y}-L_{x} R_{y}-L_{y} R_{x}\right)=0 .
\end{aligned}
$$

Then if $\alpha+\beta+\gamma+\delta \neq 0$, we obtain

$$
R_{x y+y x}-L_{x y+y x}=\left(R_{x}+L_{x}\right)\left(R_{y}-L_{y}\right)+\left(R_{y}+L_{y}\right)\left(R_{x}-L_{x}\right) .
$$

When the characteristic of $F$ is prime to six, equation (15) implies that $x x^{2}=x^{2} x[2$, p. 555] and so Postulate II is redundant.

By combining equations (8) through (13) in a different way, we obtain

$$
\begin{aligned}
& R_{x y-y x}-L_{x} L_{y}+L_{y} L_{x}-R_{y} L_{x}+R_{x} L_{y} \\
& \quad=(\alpha-\beta-\gamma+\delta)\left(L_{x y-y x}+R_{x} R_{y}-R_{y} R_{x}-L_{x} R_{y}+L_{y} R_{x}\right) \\
& \quad+(\epsilon-\eta-\sigma+\tau)\left(R_{x y-y x}-L_{x} L_{y}+L_{y} L_{x}-R_{y} L_{x}+R_{x} L_{y}\right) .
\end{aligned}
$$

Using (6) and (7), we see that (16) is equivalent to

$$
\begin{aligned}
(\alpha-\beta-\gamma+\delta) & \left(R_{x y-y x}-L_{x y-y x}-L_{x} L_{y}+L_{y} L_{x}-R_{x} R_{y}\right. \\
& \left.+R_{y} R_{x}-R_{y} L_{x}+R_{x} L_{y}+L_{x} R_{y}-L_{y} R_{x}\right)=0 .
\end{aligned}
$$

Then if $\alpha-\beta-\gamma+\delta \neq 0$, we obtain

(18) $R_{x y-y x}-L_{x y-y x}=\left(R_{x}-L_{x}\right)\left(R_{y}-L_{y}\right)-\left(R_{y}-L_{y}\right)\left(R_{x}-L_{x}\right)$.

This is a necessary and sufficient condition that the algebra $A$ be Lie-admissible $\left[2\right.$, p. 575], that is, that the algebra $A^{-}$in which the product is $[x, y]=x y-y x$ be a Lie algebra. We have proved

THEOREM 1. If $A$ is an almost left alternative algebra such that $\alpha-\beta-\gamma+\delta \neq 0$, then $A$ is Lie-admissible. 
3. Flexible algebras. In terms of right and left multiplications, the flexible law is the assumption that $R_{x} L_{x}=L_{x} R_{x}$ for every element $x$ of the algebra. Consequently, $R_{x+y} L_{x+y}=L_{x+y} R_{x+y}$, and so

$$
R_{x} L_{y}+R_{y} L_{x}=L_{x} R_{y}+L_{y} R_{x} .
$$

This may also be written in the forms

$$
R_{x} L_{y}-L_{y} R_{x}=L_{x} R_{y}-R_{y} L_{x}
$$

and

$$
y(z x)-(y z) x=(x z) y-x(z y) .
$$

Interchanging $z$ and $y,(21)$ is equivalent to

$$
R_{y x}-R_{y} R_{x}=L_{x y}-L_{y} L_{x} .
$$

From this it follows that every flexible almost left alternative algebra is almost right alternative and hence almost alternative.

Using (6), (7), and (20), identity (8) for almost left alternative algebras can be written in the form

$$
\begin{aligned}
R_{x y}-R_{x} R_{y}= & (\alpha-1)\left(R_{x} R_{y}+L_{x} L_{y}\right)+\beta\left(R_{y} R_{x}+L_{y} L_{x}\right) \\
& +(1-\alpha-\beta)\left(R_{x} L_{y}+R_{y} L_{x}\right) \\
& +(\gamma-\delta)\left(L_{x} R_{y}-R_{y} L_{x}\right) .
\end{aligned}
$$

If $y=x$,

$$
R_{x x}-R_{x} R_{x}=(\alpha+\beta-1)\left(R_{x}-L_{x}\right)^{2}
$$

so that $A$ is alternative if and only if $\alpha+\beta=1$ or $\left(R_{x}-L_{x}\right)^{2}=0$.

Theorem 2. Let $A$ be a flexible almost left alternative algebra which is not alternative. Then $(\alpha-\beta+\gamma-\delta)(\alpha+\beta-1)=\beta$.

Proof. Take $y=x$ in (10) and use (6), (7), and the flexible law to obtain $L_{x}^{2}=(\alpha+\gamma) L_{x x}-(\beta+\delta) R_{x x}+\delta R_{x}^{2}+(1-\alpha-\beta-\gamma) L_{x}^{2}+2 \beta R_{x} L_{x}$. From (22) it follows that $L_{x x}=R_{x x}-R_{x}^{2}+L_{x}^{2}$. Then $(\alpha-\beta+\gamma-\delta)$ . $\left(R_{x x}-R_{x}^{2}\right)=\beta\left(R_{x}^{2}+L_{x}^{2}-2 R_{x} L_{x}\right)$. Combining with (24) we obtain $[(\alpha-\beta+\gamma-\delta)(\alpha+\beta-1)-\beta] \cdot\left(R_{x}-L_{x}\right)^{2}=0$. Since $A$ is assumed to be not alternative, $\left(R_{x}-L_{x}\right)^{2} \neq 0$ and the identity follows.

Because of flexibility, $R_{x}$ commutes with $L_{x}$. For this reason, any algebra which is flexible and shrinkable of level one satisfies the relation $R_{x} R_{x x}=R_{x x} R_{x}$, since $R_{x x}$ is expressible in terms of $R_{x}$ and $L_{x}$. This relation and that of flexibility are the postulates for noncommutative Jordan algebras, and as observed by Schafer in [3] such algebras with characteristic not two are Jordan-admissible and power-associative. 
Theorem 3. Every flexible almost left alternative algebra $A$ with $\alpha+\beta \neq 3 / 4$ is quasiequivalent in a scalar extension $K$ of $F$ to an alternative algebra $B=A_{K}(\lambda)$.

Proof. Let $K=F(\xi)$ where $\xi^{2}=4 \alpha+4 \beta-3$ and let $\lambda=(\xi+1) / 2$. Since $\alpha+\beta \neq 3 / 4, \xi \neq 0$. Thus $\lambda$ is a quantity of $K$ different from $1 / 2$. Form $A_{K}(\lambda)=B$. Since $A$ is flexible, so is $A_{K}$. As shown in [2], $A_{K}(\lambda)$ is also flexible. Hence to show $B$ alternative, it is sufficient to show $R_{x x}^{\prime}=R_{x}^{\prime 2}$, where $R_{x}^{\prime}$ is a right multiplication of $B$. Since $R_{x}^{\prime}$ $=\lambda R_{x}+(1-\lambda) L_{x}, R_{x x}^{\prime}=\lambda R_{x x}+(1-\lambda) L_{x x}=R_{x x}+(1-\lambda)\left(L_{x}^{2}-R_{x}^{2}\right)$ using (22); and $R_{x}^{\prime 2}=\lambda^{2} R_{x}^{2}+2 \lambda(1-\lambda) R_{x} L_{x}+(1-\lambda)^{2} L_{x}^{2}$.

Now $1-\lambda=(1-\xi) / 2$ and $\alpha+\beta-1=\left(\xi^{2}-1\right) / 4=-\lambda(1-\lambda)$. Thus (24) becomes $R_{x x}=R_{x}^{2}-\lambda(1-\lambda)\left(R_{x}-L_{x}\right)^{2}$, so that $R_{x x}^{\prime}=R_{x}^{2}-\lambda(1-\lambda)$ $\cdot\left(R_{x}-L_{x}\right)^{2}+(1-\lambda)\left(L_{x}^{2}-R_{x}^{2}\right)=\lambda^{2} R_{x}^{2}+2 \lambda(1-\lambda) R_{x} L_{x}+(1-\lambda)^{2} L_{x}^{2}=R_{x}^{\prime 2}$.

Corollary. A flexible almost left alternative algebra $A$ with $\alpha+\beta$ $\neq 3 / 4$ over a field $F$ of charactristic prime to 6 is quasiassociative if and only if $A$ is Lie-admissible.

Proof. It is shown in [2] that an alternative algebra over a field of characteristic not 2 or 3 is associative if and only if it is Lie-admissible; and furthermore that $B$ is Lie-admissible if and only if $B(\mu)$ is Lie-admissible. Now $A_{K}=B(\mu)$ where $\mu=\lambda /(2 \lambda-1)$ and $A_{K}$ is Lieadmissible if and only if $A$ is Lie-admissible.

\section{REFERENCES}

1. A. A. Albert, Almost alternative algebras, Portugaliae Mathematica vol. 8 (1949) pp. 23-36.

2. - Power-associative rings, Trans. Amer. Math. Soc. vol. 64 (1948) pp. $552-593$.

3. R. D. Schafer, Noncommutative Jordan algebras of characteristic 0, Proc. Amer. Math. Soc. vol. 6 (1955) pp. 472-475.

The University of Chicago 